

\title{
Ciencia Nicolaita
}

\section{Estudios de repetibilidad y reproducibilidad de métodos no destructivos en maderas angiospermas}

\author{
Javier Ramón Sotomayor Castellanos, René Navarrete Padilla
}

Para citar este artículo: Sotomayor Castellanos Ramón Javier, Navarrete Padilla René. 2021. Estudios de repetibilidad y reproducibilidad de métodos no destructivos en maderas angiospermas. Ciencia Nicolaita, número 82, 89-104. DOI: https://doi.org/10.35830/cn.vi82.533



Términos y condiciones de acceso y uso en https://www.cic.cn.umich.mx/cn/about/privacy 


\title{
Estudios de repetibilidad y reproducibilidad de métodos no destructivos en maderas angiospermas
}

\author{
Javier Ramón Sotomayor Castellanos ${ }^{{ }^{*}}$, René Navarrete Padilla ${ }^{2}$
}

${ }^{1}$ Universidad Michoacana de San Nicolás de Hidalgo, Facultad de Ingeniería en Tecnología de la Madera

${ }^{2}$ Universidad de Guanajuato, División de Arquitectura, Arte y Diseńo

\section{Historial del ARTí́culo}

Recibido: 23 de octubre de 2020

Aceptado: 13 de marzo de 2021

\section{RESUMEN}

Los estudios de repetibilidad y reproducibilidad (R\&R) de las características mecánicas de la madera pueden garantizar la validez de los datos experimentales empleados en la caracterización y cálculo ingenieril. El objetivo de la investigación fue verificar que un estudio de $R \& R$ de la velocidad de onda, determinada con pruebas no destructivas, explica las interacciones entre los instrumentos, operadores y probetas. La primera etapa experimental consistió en el análisis de los resultados de las pruebas de ondas de esfuerzo y de ultrasonido realizadas en probetas de Fraxinus americana y Albizia plurijuga. En la segunda etapa se analizaron las variaciones de los resultados de la velocidad de onda. La investigación confirma la conveniencia de los estudios de R\&R para la validación de las mediciones de velocidad de onda con los dos métodos citados. La variabilidad de la velocidad de onda es causada principalmente por el instrumento de medición, pero esta es pequeña en comparación con la variabilidad entre las especies analizadas. Las magnitudes de las interacciones son particulares a cada caso de estudio.

Palabras ClavE: velocidad de onda, ondas de esfuerzo, ultrasonido, Fraxinus americana, Albizia plurijuga.

\section{ABstract}

The repeatability and reproducibility $(\mathrm{R} \& \mathrm{R})$ studies of the mechanical characteristics of wood can guarantee the validity of the experimental data used in the characterization and engineering calculation. The objective of the investigation was to verify that an R\&R study of the wave velocity, determined with non-destructive tests, explains the interactions between the instruments, operators and specimens. The first experimental stage consisted of the analysis of the results of the stress wave and ultrasound tests carried out on specimens of Fraxinus americana and Albizia plurijuga. In the second stage, the variations of the wave velocity results were analyzed. The research confirms the suitability of R\&R studies for the validation of wave velocity measurements with the two cited methods. The variability of the wave speed is caused mainly by the measuring instrument, but this is small compared to the variability between the analyzed species. The magnitudes of the interactions are particular to each case study.

KEYWORDS: wave velocity, stress waves, ultrasound, Fraxinus americana, Albizia plurijuga.

CORRESPONDENCIA DE AUTOR: madera999@yahoo.com

Ciencia Nicolaita Copyright, Universidad Michoacana de San Nicolás de Hidalgo. 


\section{Introducción}

Los estudios de repetibilidad y reproducibilidad (R\&R) son los más usados en el análisis de sistemas de medición (Gong et al., 2015, Ha et al., 2017). La repetibilidad se define como la variación en las mediciones obtenidas con un medidor utilizado varias veces por un operador, mientras se mide una característica en una parte. La reproducibilidad se define como la variación en el promedio de las mediciones realizadas por diferentes operadores que usan el mismo indicador al medir una característica en una parte (Wang y Chien, 2010; Saikaew, 2018). En otras palabras, la repetibilidad se refiere a la variación de mediciones repetidas en un mensurando por un operador que usa el mismo dispositivo de medición. Por su parte la reproducibilidad se relaciona con la variación de los promedios de medición entre varios dispositivos de medición, distintos procedimientos y/o diferentes operadores (Mohamed et al., 2018; Mendes et al., 2019). Los principales componentes de los estudios de R\&R son muestras al azar de piezas del proceso, las cuales son medidas por operadores escogidos aleatoriamente varias veces con uno o varios dispositivos de medición ( $\mathrm{Li}$ y Al-Refaie, 2008; Zanobini et al., 2016).

Los estudios de R\&R reportados en la literatura están encaminados al control estadístico de la calidad, así como al análisis de la estabilidad de sistemas de medición. Esta técnica tiene múltiples enfoques y amplias aplicaciones (Senvar y First, 2010; Gómez, 2013; Peruchi et al., 2013; González y Falcón, 2015; Tiplica et al., 2015; López et al., 2018). En este sentido, los estudios de R\&R se orientan a la evaluación de los sistemas de medición en ambientes industriales donde las características de los productos o piezas por evaluar pueden ser controlables. Las ventajas de los estudios de R\&R por el método del análisis de varianza son su flexibilidad para estudiar cual- quier arreglo o estructura experimental, el uso de varianzas como parámetros de comparación, el manejo de mayor información de los datos experimentales y la identificación de la interacción entre la repetibilidad y la reproducibilidad (González y Falcón, 2015; Botero et al., 2007). De esta manera el análisis de varianza se aplica para detectar estas fuentes de variación.

Para estudiar procesos donde intervienen métodos no destructivos, se trabaja bajo los supuestos de estabilidad temporal de los mensurados y de la solidez entre mediciones. Es decir, las propiedades físicas de los objetos no se ven modificadas por el proceso de medición. En este contexto, la repetibilidad se puede definir como la varianza de las mediciones obtenidas al medir las características de un dispositivo de medición. Por su parte, la reproducibilidad es la varianza del medio de las mediciones obtenidas por diferentes operadores, al medir las mismas características de la misma pieza, con el mismo dispositivo de medición (Senol, 2004; De Mast y Trip, 2005). Así, los estudios de R\&R proporcionan criterios para estimar dos componentes independientes de la varianza de medición.

Los estudios de $\mathrm{R} \& \mathrm{R}$ de características mecánicas de la madera, son importantes para garantizar la validez de los datos experimentales empleados en cálculo ingenieril y son valiosos para contrastar los datos de diferentes investigaciones (Shi et al., 2014). Sin embargo, con excepción de Yu et al. (2017), no se identificaron trabajos aplicados a la caracterización de maderas por métodos no destructivos. Existe evidencia empírica de variabilidad en las características físicas de la madera entre especies y al interior de una especie (De Oliveira y Sales, 2006). En este contexto, la evaluación de mediciones de velocidades de onda en la madera, aplicando un estudio de $R \& R$, requiere un enfoque diferente, dado que las probetas o piezas de madera a observar son de origen natural y resultado de la 
fisiología particular a cada especie y de las condiciones de crecimiento del árbol.

\section{Pruebas de ondas de esfuerzo y ultrasonido}

La velocidad de onda en la madera está documentada con pruebas de ondas de esfuerzo (Lin y Wu, 2013; Yu et al., 2017; Nasir et al., 2019) y de ultrasonido (De Oliveira y Sales, 2006; Del Menezzi et al., 2014; Gonçalves et al., 2014). La literatura acerca de la caracterización mecánica de la madera de especies mexicanas también informa sobre datos obtenidos con estos dos métodos no destructivos (Sotomayor, 2014; Sotomayor, 2015). Empero, no existen antecedentes de estudios de R\&R comparando estos métodos y midiendo maderas mexicanas.

Las magnitudes de las velocidades de onda varían entre especies y según el método empleado para su caracterización (Hasegawa et al., 2011; Del Menezzi et al., 2014). De aquí surgen las siguientes interrogaciones: ¡es la variabilidad del instrumento de medición pequeña en comparación con la variabilidad de la especie? y ¿cuánto de la variabilidad de la velocidad de onda medida en una probeta es causada por el aparato y/o por el operador?

Para responder a estas preguntas se plantea la siguiente hipótesis de investigación: el estudio de $\mathrm{R} \& \mathrm{R}$ de las velocidades de onda determinadas con ondas de esfuerzo y con ultrasonido en una misma especie explica las fuentes de variabilidad y las interacciones entre los instrumentos, operadores y probetas. Esta hipótesis está restringida a los resultados experimentales de muestras homogéneas y estadísticamente representativas de las maderas estudiadas, así como a las condiciones particulares de la metodología empleada.

El objetivo de la investigación fue verificar experimentalmente la hipótesis de investigación efectuando estudios de $R \& R$ de las velocidades de onda derivadas de pruebas no destructivas. Para esto se realizaron pruebas de ondas de esfuerzo y de ultrasonido en probetas de madera de Fraxinus americana y Albizia plurijuga y con las mediciones se realizaron sus análisis estadísticos. El alcance y límite de la investigación es informar sobre la conveniencia de los estudios $\mathrm{R} \& \mathrm{R}$ para la validación de las mediciones de velocidades de onda con los dos métodos citados. Los resultados pretenden contribuir en la aplicación de los estudios R\&R en ciencias y tecnología de la madera.

\section{Materiales y métodos}

La estrategia experimental asume dos etapas: la primera es el análisis de los resultados de las velocidades de onda, derivados de pruebas de ondas de esfuerzo y de ultrasonido que se realizaron en dos especies angiospermas: Fraxinus americana L. y Albizia plurijuga (Standl.) Britton y Rose. Esta etapa tiene como objetivo validar la homogeneidad y la representatividad de las muestras de las maderas en estudio. La segunda etapa se refiere a los estudios de $R \& R$ de las variaciones de los resultados de las velocidades de onda. Su objetivo es construir tablas de repetibilidad y reproducibilidad, así como de su análisis de varianza. Los cálculos y análisis estadísticos fueron realizados con el programa Statgraphics ${ }^{\circledast}$, siguiendo las recomendaciones de Gutiérrez y De la Vara (2009) y Gutiérrez y De la Vara (2013).

Se recolectaron piezas de madera aserrada de $F$. americana y $A$. plurijuga en empresas de transformación de productos forestales en el estado de Michoacán, México. La madera no contenía anomalías estructurales ni defectos de crecimiento y con ella se prepararon 40 probetas con una sección transversal de 0,02 $\mathrm{m}$ de ancho, 0,02 $\mathrm{m}$ de espesor y de 0,4 $\mathrm{m}$ de largo 
de acuerdo con la norma ISO 3129: 2012 (International Organization for Standardization, 2012). Las probetas se almacenaron durante 24 meses en una cámara de acondicionamiento con una temperatura de $20{ }^{\circ} \mathrm{C}\left( \pm 1{ }^{\circ} \mathrm{C}\right)$ y una humedad relativa del aire de $65 \%( \pm 5 \%)$, hasta que su peso fue constante y la madera alcanzó su contenido de humedad en equilibrio. El presente estudio forma parte de la línea de investigación de caracterización mecánica de maderas mexicanas, desarrollada en la Facultad de Ingeniería en Tecnología de la Madera, de la Universidad Michoacana de San Nicolás de Hidalgo, en Morelia, Michoacán, México. Por este motivo el material experimental y las velocidades de onda son los reportados por Sotomayor y Ávila (2020a) y Sotomayor y Ávila (2020b).

La densidad de la madera se determinó con la relación peso/volumen de acuerdo con la norma ISO 13061-2:2014 (International Organization for Standardization, 2014a). El contenido de humedad se determinó por el método de diferencia de pesos de acuerdo con la norma ISO 13061-1:2014 (International Organization for Standardization, 2014b). Ambos parámetros se determinaron en 40 probetas adicionales obtenidas de los mismos lotes de madera para las pruebas de ondas de esfuerzo y ultrasonido, con dimensiones de $0,02 \mathrm{~m} \times 0,02 \mathrm{~m}$ de sección y $0,06 \mathrm{~m}$ de largo. Para que los errores aleatorios propios de estas mediciones no influyan en los resultados, los pesos fueron medidos por operadores capacitados con una balanza digital con precisión de $0,1 \mathrm{~g}$ y las dimensiones de las probetas fueron medidas con un calibrador con precisión de $0,00001 \mathrm{~m}$.

Las pruebas de ondas de esfuerzo (Sotomayor et al., 2020) consistieron en suministrar un impacto en la dirección longitudinal en un extremo de la probeta. Con un acelerómetro emisor, posicionado en el aparato Metriguard ${ }^{\top}$ con frecuencia de emisión de $44 \mathrm{kHz}$ y precisión de
0,000001 s (Figura 1a), registrándose el tiempo inicial. En el otro extremo de la probeta, en un segundo acelerador receptor, se registró el tiempo de transmisión de las ondas de esfuerzo. Con este valor, dividido por la distancia entre los acelerómetros, se definió la velocidad de la onda de esfuerzo $\left(\mathrm{v}_{\mathrm{oe}}\right)$.

Las pruebas de ultrasonido (Sotomayor, 2020) consistieron en suministrar un impulso ultrasónico en transmisión directa a lo largo de la probeta con el aparato Sylvatest ${ }^{\oplus}$ con frecuencia de emisión de $22 \mathrm{kHz}$ y precisión de 0,000001 s (Figura 1b). De esta forma se midió el tiempo de transmisión de la onda en la dirección longitudinal. Con la longitud de las probetas y el tiempo de transmisión del ultrasonido, se calculó la velocidad de onda del ultrasonido $\left(\mathrm{v}_{\mathrm{us}}\right)$.

\section{Diseño experimental}

La unidad experimental consistió en dos grupos homogéneos de 40 probetas cada uno de maderas de $F$ americana y $A$. plurijuga seleccionadas de manera aleatoria. El instrumento de medición (aparatos de ondas de esfuerzo versus ultrasonido) se considera el factor de variación. Los pesos, las dimensiones de las probetas, así como el contenido de humedad de la madera y su densidad, son factores fijos y controlables. Las variables de entrada son las velocidades de onda medidas con ondas de esfuerzo y las variables de salida son las medidas con ultrasonido. Se realizó un experimento de un solo factor de variación (tipo de prueba), el cual consistió en el análisis de las velocidades de onda ( $\mathrm{v}_{\text {oe }}$ versus $\mathrm{v}_{\mathrm{us}}$ ) con dos niveles (F. americana y $A$. plurijuga).

Para cada par de muestras independientes (ondas de esfuerzo versus ultrasonido) se calcularon las medias $(\mu)$, las desviaciones estándar $(\sigma)$ y los coeficientes de variación $(\mathrm{CV}=\sigma / \mu)$. Se calcularon los sesgos (S) y apuntamientos (A) 
derivados de pruebas de normalidad. El criterio de demarcación para considerar normal la distribución de una muestra fue: $[-2<S<+2,-2<$ A $<+2]$. Se verificaron las igualdades de varianzas y se practicaron análisis de varianzas. Para todas las pruebas el nivel de confiabilidad fue de $95 \%$ ( $\alpha$ $=0,05)$. Por lo tanto, el criterio de demarcación fue aceptar una diferencia estadísticamente significativa para valores $P_{(\alpha=0,05)} \leq 0,05$. Se verificó la hipótesis nula $\mathrm{H}_{0}: \mu_{1}=\mu_{2}$, donde: $\mathrm{H}_{0}=$ Hipótesis nula (el tipo de prueba no influye en el parámetro calculado); $\mu_{1}=$ Medias de las velocidades de onda con ondas de esfuerzo; $\mu_{2}=$ Medias de las velocidades de onda por ultrasonido. Se calculó el tamaño de la muestra para un error aceptable de 0,05 de acuerdo con Gutiérrez y De la Vara (2009).

\section{Estudios de repetibilidad y reproducibilidad}

Se realizaron dos estudios cruzados incluyendo interacción de operador por probeta (repetibilidad y reproducibilidad), uno para para cada especie estudiada ( $F$. americana y $A$. plurijuga). Se analizaron datos empíricos de las velocidades de onda medidas con dos instrumentos (medidores de ondas de esfuerzo y de ultrasonido). Con cada instrumento dos operadores realizaron dos mediciones aleatorias en 20 probetas (partes) en la dirección longitudinal (Figuras 1a y 1b). Estas mediciones se analizaron como variables aleatorias e independientes. Se realizaron análisis de varianza $\left(\sigma^{2}\right)$ entre los instrumentos y partes. El nivel de confiabilidad fue de $95 \%$. Por lo tanto, el criterio de demarcación fue aceptar que no existe interacción significativa para valores de $\mathrm{P}_{(\alpha=0,05)}>0,05$.

Las fuentes de variabilidad que se evaluaron en el estudio de R\&R se definieron de acuerdo con Gutiérrez y De la Vara (2013): $\sigma_{\text {probetas }}^{2}=$ Variabilidad atribuible a las probetas

$\sigma_{\text {instrumentos }}^{2}=$ Variabilidad de los instrumentos de medición

$\sigma_{\text {operadores }}^{2}=$ Variabilidad de los operadores

$\sigma_{\text {operadores x probetas }}^{2}=$ Variabilidad por la interacción entre operadores y probetas

De tal forma que la variabilidad total observada fue:

$\sigma_{\text {total }}^{2}=\sigma_{\text {probetas }}^{2}+\sigma_{\text {operadores }}^{2}+\sigma_{\text {operadores x probetas }}^{2}+\sigma_{\text {instrumentos }}^{2}$

Considerando que:

\begin{tabular}{|l|l|}
\hline$\sigma_{\text {repetibilidad }}^{2}=\sigma_{\text {instrumentos }}^{2}$ & (2) \\
\hline$\sigma_{\text {reproductibilidad }}^{2}=\sigma_{\text {operadores }}^{2}+\sigma_{\text {operadores x probetas }}^{2}$ & (3) \\
\hline
\end{tabular}

La variabilidad R\&R se definió como:

$\sigma_{\mathrm{R} \& \mathrm{R}}^{2}=\sigma_{\text {repetibilidad }}^{2}+\sigma_{\text {reproductibilidad }}^{2}$

De los datos del estudio de R\&R se calcularon las sumas de cuadrados (SC) correspondientes a cada componente de variación dado en la ecuación (1), mismas que cumplen la relación:

$\mathrm{SC}_{\text {total }}=\mathrm{SC}_{\text {probetas }}+\mathrm{SC}_{\text {operadores }}+\mathrm{SC}_{\text {operadores x probetas }}+\mathrm{SC}_{\text {instrumentos }}$

La suma de cuadrados $\mathrm{SC}_{\text {instrumentos }}$ corresponde a la suma de cuadrados del error $\mathrm{SC}_{\text {error }}$.

Al dividir cada suma de cuadrados por sus grados de libertad se obtuvieron los cuadrados medios (CM). Con estos parámetros se construyeron pruebas estadísticas para verificar diferencias entre las partes, entre los operadores y el efecto de interacción operadores $x$ probetas. Si se definen $\mathrm{p}=$ probetas, $\mathrm{o}=$ operadores $\mathrm{y} \mathrm{t}=$ pruebas, los grados de libertad correspondientes a cada suma de cuadrados de la ecuación (5) fueron, respectivamente: 


\begin{tabular}{|l|c|}
\hline $\mathrm{GL}_{\text {probetas }}=(\mathrm{p}-1)$ & $(6)$ \\
\hline $\mathrm{GL}_{\text {operadores }}=(\mathrm{o}-1)$ & $(7)$ \\
\hline $\mathrm{GL}_{\text {operadores } \times \text { probetas }}=[(\mathrm{o}-1) \times(\mathrm{p}-1)]$ & $(8)$ \\
\hline $\mathrm{GL}_{\text {instrumentos }}=[\mathrm{p} \times \mathrm{o} \times(\mathrm{t}-1)]$ & $(9)$ \\
\hline $\mathrm{GL}_{\text {total }}=(\mathrm{p}-1)+(\mathrm{o}-1)+[(\mathrm{o}-1) \times(\mathrm{p}-1)]+[\mathrm{p} \times \mathrm{O} \times(\mathrm{t}-1)]$ & $(10)$ \\
\hline
\end{tabular}

Además de los valores esperados de los cuadrados medios, se dedujo que los estimadores de los componentes de varianza para cada caso estuvieron dados por:

\begin{tabular}{|l|l|}
\hline$\sigma_{\text {instrumentos }}^{2}=C M_{\text {error }}$ & (11) \\
\hline$\sigma_{\text {probetas }}^{2}=\frac{C M_{\text {probetas }}-C M_{\text {operadores } \times \text { probetas }}}{\mathrm{t} \times 0}$ & (12) \\
\hline$\sigma_{\text {operadores }}^{2}=\frac{C M_{\text {operadores }}-C M_{\text {operadores } \times \text { probetas }}}{\mathrm{t} \times \mathrm{p}}$ & (13) \\
\hline$\sigma_{\text {operadores } \times \text { partes }}^{2}=\frac{\mathrm{CM}_{\text {operadores } \times \text { probetas }}-\mathrm{CM}_{\text {error }}}{\mathrm{t}}$ & \\
\hline
\end{tabular}

De esta manera se obtuvo la repetibilidad, la reproducibilidad y el error de medición, los cuales, para su interpretación, se expresan como porcentajes de la variación total.

\section{Resultados y discusión}

El contenido de humedad para $F$. americana fue de $10 \%(\mathrm{CV}=6 \%)$ y para $A$. plurijuga de $12 \%$ $(\mathrm{CV}=5 \%)$. La densidad de la madera fue para $F$. americana de $654 \mathrm{~kg} \mathrm{~m}^{-3}(\mathrm{CV}=6 \%)$ y para A. plurijuga fue de $830 \mathrm{~kg} \mathrm{~m}^{-3}(\mathrm{CV}=3 \%)$. Estos resultados coinciden con los reportados por Sotomayor y Ávila (2020a) y Sotomayor y Ávila (2020b) para ambas especies.
Pruebas de ondas de esfuerzo y de ultrasonido

La Tabla 1 presenta los resultados de las pruebas de ondas de esfuerzo y ultrasonido. Las velocidades de onda del ultrasonido de $F$. americana son en promedio $13 \%$ mayores que las correspondientes a las de ondas de esfuerzo. Las correspondientes para $A$. plurijuga son también $17 \%$ mayores que las medidas por ondas de esfuerzo. Para $F$. americana, sus coeficientes de variación de ondas de esfuerzo y ultrasonido son en promedio 4,75\%. Para $A$. plurijuga son mayores y en promedio $7,98 \%$.

Para ambas especies, las pruebas de normalidad indican que las muestras están uniformemente repartidas. Asimismo, las varianzas verificaron su igualdad. Finalmente, los análisis de varianza indican diferencias estadísticamente significativas entre las velocidades de onda según el instrumento de medición. Empero, las velocidades de onda son grupos homogéneos al interior de cada especie según el tipo de instrumento de medición. Para cada grupo que se midió, el tamaño de muestra calculado es menor y suficiente respecto al número de pruebas medidas. Las Figuras 2 y 3 presentan la convergencia del tamaño de la muestra (número de probetas necesarias) en función del error aceptable para $F$. americana y $A$. plurijuga respectivamente (Tabla 1). Los estudios de R\&R de un sistema de medición (cruzados), igual que otros procedimientos de análisis de sistemas de medición, son experimentos diseñados. Para que los resultados sean válidos, la aleatorización y el muestreo representativo son esenciales.

Estos resultados verifican la homogeneidad de las muestras, la diferenciación de las velocidades de onda medidas con ondas de esfuerzo, especificadas como variables de entrada y las medidas con ultrasonido catalogadas como variables de salida, de tal forma que se pueden analizar como 
Tabla 1. Resultados de las pruebas de ondas de esfuerzo y ultrasonido

\begin{tabular}{|c|c|c|c|c|}
\hline \multirow{2}{*}{$\begin{array}{l}\text { Instrumento de medición } \\
\text { Parámetro }\end{array}$} & \multicolumn{2}{|c|}{ Ondas de esfuerzo } & \multicolumn{2}{|c|}{ Ultrasonido } \\
\hline & $\mathrm{v}_{\mathrm{oe}}$ & $\mathrm{v}_{\mathrm{oe}}$ & $\mathrm{v}_{\mathrm{us}}$ & $\mathrm{v}_{\mathrm{us}}$ \\
\hline Unidades & $\left(\mathrm{m} \mathrm{s}^{-1}\right)$ & $\left(\mathrm{m} \mathrm{s}^{-1}\right)$ & $\left(\mathrm{m} \mathrm{s}^{-1}\right)$ & $\left(\mathrm{m} \mathrm{s}^{-1}\right)$ \\
\hline Operador & 1 & 2 & 1 & 2 \\
\hline Especie & \multicolumn{4}{|c|}{ Fraxinus americana } \\
\hline Media & 4008 & 4025 & 4498 & 4601 \\
\hline Desviación estándar & 183 & 197 & 205 & 231 \\
\hline Coeficiente de Variación & 5 & 5 & 5 & 5 \\
\hline Mínimo & 3704 & 3636 & 4124 & 4167 \\
\hline Máximo & 4396 & 4396 & 4878 & 5000 \\
\hline Sesgo & 0,425 & $-0,204$ & $-0,149$ & 0,187 \\
\hline Apuntamiento & $-0,089$ & 0,400 & $-0,777$ & $-0,458$ \\
\hline \multirow[t]{2}{*}{ Tamaño de la muestra } & 3 & 4 & 3 & 4 \\
\hline & \multicolumn{4}{|c|}{ Valor $\mathrm{P}_{(\alpha=0,05)}$} \\
\hline Verificación de varianza & \multicolumn{4}{|l|}{$0,672^{*}$} \\
\hline Análisis de varianza & \multicolumn{4}{|l|}{$<0,001^{\#}$} \\
\hline Grupos homogéneos & $\mathrm{X}$ & $\mathrm{X}$ & & \\
\hline Grupos homogéneos & & & $\mathrm{X}$ & $\mathrm{X}$ \\
\hline Especie & \multicolumn{4}{|c|}{ Albizia plurijuga } \\
\hline Media & 4071 & 4040 & 4775 & 4689 \\
\hline Desviación estándar & 277 & 259 & 440 & 444 \\
\hline Coeficiente de Variación & 7 & 6 & 9 & 10 \\
\hline Mínimo & 3604 & 3509 & 4000 & 4211 \\
\hline Máximo & 4545 & 4444 & 5556 & 5479 \\
\hline Sesgo & $-0,026$ & $-0,095$ & 0,184 & 0,599 \\
\hline Apuntamiento & $-1,279$ & $-0,930$ & $-0,447$ & $-0,814$ \\
\hline \multirow[t]{2}{*}{ Tamaño de la muestra } & 7 & 10 & 15 & 18 \\
\hline & \multicolumn{4}{|c|}{ Valor $\mathrm{P}_{(\alpha=0,05)}$} \\
\hline Verificación de varianza & \multicolumn{4}{|c|}{$0,115^{*}$} \\
\hline Análisis de varianza & \multicolumn{4}{|c|}{$<0,001^{\#}$} \\
\hline Grupos homogéneos & $\mathrm{X}$ & $\mathrm{X}$ & & \\
\hline Grupos homogéneos & & & $\mathrm{X}$ & $\mathrm{X}$ \\
\hline
\end{tabular}

$\mathrm{v}=$ Velocidad de onda; oe $=$ Ondas de esfuerzo; $\mathrm{us}=$ Ultrasonido; Coeficiente de variación en porciento; ${ }^{*} \mathrm{P}_{(\alpha=0,05)}>0,05=$ No existe diferencia significativa; $\# \mathrm{P}_{(\alpha=0,05)}<0,05=$ Sí existe diferencia significativa; Filas compartiendo X son grupos homogéneos; Tamaño de la muestra para un error de $0,05 \%$. Fuente: elaboración propia. 




Figura 1. Pruebas de: a) ondas de esfuerzo; b) ultrasonido (Sotomayor et al., 2011).

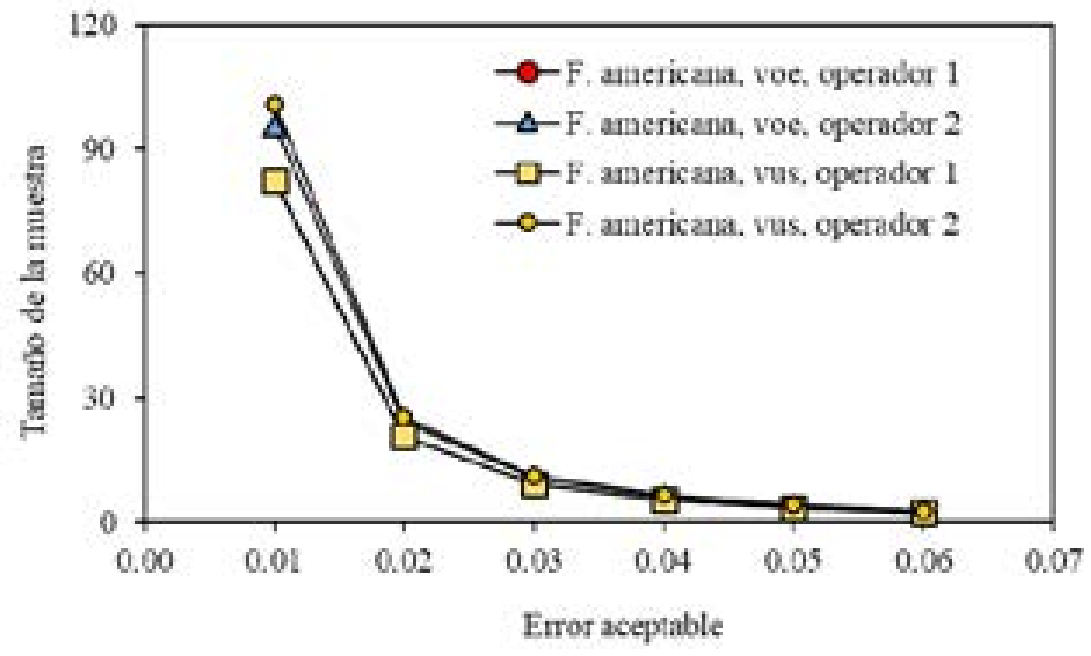

Figura 2. Tamaño de la muestra en función del error aceptable de $F$. americana.

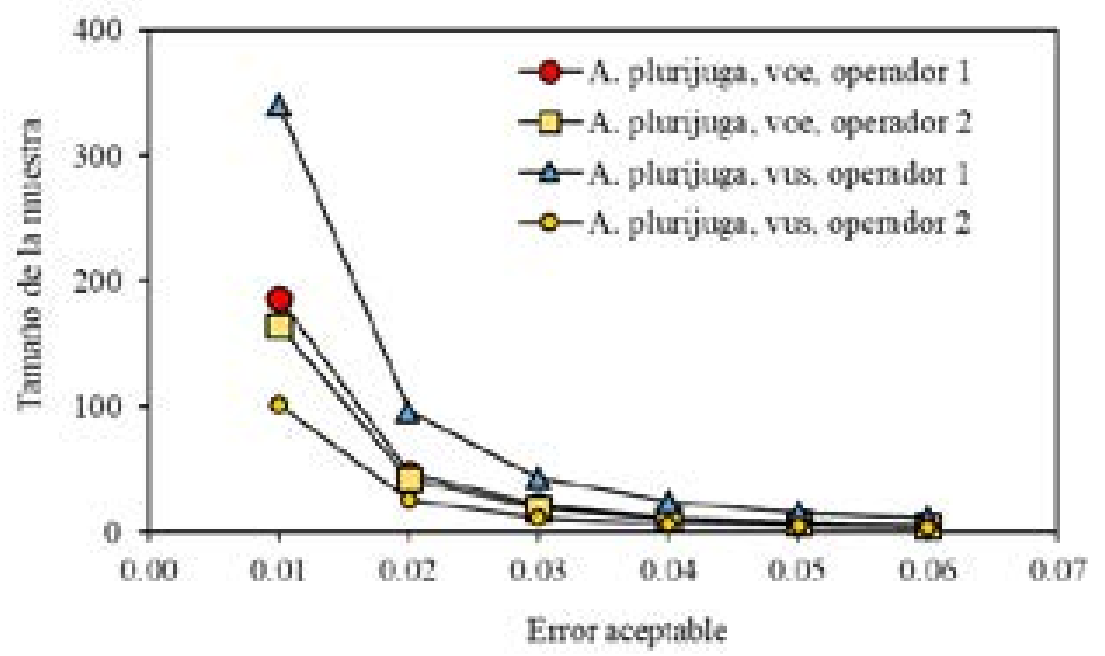

Figura 3. Tamaño de la muestra en función del error aceptable de $A$. plurijuga. 
variables aleatorias e independientes en los estudios de R\&R. El alcance de este enfoque es limitado para cuantificar la exactitud de las mediciones. Esto sucede por el hecho de que no se puede determinar un valor medio para la velocidad de onda de una especie de madera y por consiguiente es difícil cuantificar la diferencia entre un valor experimental promedio y un valor de referencia. Caso particular son los valores de las características mecánicas tabuladas en los códigos y reglamentos de construcción con madera. En cambio, el coeficiente de variación puede ser un indicador de la precisión del sistema de medición cuando la misma probeta se mide repetidamente con el mismo instrumento. Además, la repetibilidad y reproducibilidad son componentes de un mismo sistema de medición.

En la investigación, la estrategia experimental se orientó hacia el análisis de resultados especie por especie. De acuerdo con el paradigma contemporáneo que rige en investigación e ingeniería de la madera, es necesario caracterizar el comportamiento mecánico de ésta con un enfoque de experimentación de caso por caso de una especie en particular. Cada procedimiento debe estar referido a las variables de referencia de las condiciones de ensayo, por ejemplo, la densidad y el contenido de humedad de la madera y con datos derivados de la observación de un tamaño de muestra estadísticamente representativo. Una vez que se tienen las observaciones integrantes y la estadística, se pueden proponer tendencias en el comportamiento general para una especie en específico y/o por agrupamiento de varias de ellas que denoten una tendencia similar (Sotomayor y Correa, 2016).

\section{Estudios de repetibilidad y reproducibilidad}

La Tabla 2 presenta los resultados de los estudios de R\&R y la Tabla 3 presenta los resulta- dos del análisis de varianza. Los resultados se presentan de manera independiente para cada una de las dos especies. Así, se consideran dos casos diferentes de estudio, pero con mediciones equivalentes para su comparación. Las tablas se construyeron utilizando las ecuaciones (1) a (14).

El estudio de R\&R de $F$. americana indica que el $81 \%$ de la varianza se debe a la reproducibilidad que corresponde a diferencias entre operadores y probetas, en tanto que $16 \%$ se debe a la repetibilidad que corresponde a los instrumentos (Tabla 2) y el 3\% corresponde a la fuente de variación R\&R. Para esta misma especie, los resultados presentan la misma tendencia. Los datos de la Tabla 3 indican que, entre instrumentos, sí existe contribución significativa al error de medición dado que su valor es $\mathrm{P}_{(\alpha=0,05)}<0,05$. En cambio, las probetas y la interacción de instrumentos y probetas no contribuyen de manera significativa al error de medición dado su valor $P_{(\alpha=0,05)}>0,05$. Aquí la fuente residual corresponde a la repetibilidad.

Para $A$. plurijuga, el estudio de $\mathrm{R} \& \mathrm{R}$ indica que el $44 \%$ de la varianza total se debe a la reproducibilidad que corresponde a diferencias entre los instrumentos, en tanto que $56 \%$ se debe a la repetibilidad que corresponde a operadores y probetas (Tabla 2). Como consecuencia, en este caso el factor R\&R es nulo. Para esta misma especie, la Tabla 3 indica que sí existe interacción significativa entre instrumentos y para las probetas $\left(P_{(\alpha=0,05)}<0,05\right)$. En cambio para la combinación de instrumentos y probetas no existe interacciones significativas $\left(\mathrm{P}_{(\alpha=0,05)}>0,05\right)$. De manera análoga al estudio $\mathrm{R} \& \mathrm{R}$ de $F$. americana, la fuente residual corresponde a la repetibilidad.

La repetibilidad representa la variación causada por los instrumentos de medición. En esta investigación se trata de la medición de probetas de una misma especie (F. americana y solamente esta especie, o en su caso, las probetas de $A$. plu- 
Tabla 2. Resultados de los estudios de repetibilidad y reproducibilidad

\begin{tabular}{|c|c|c|c|c|c|}
\hline \multirow[t]{2}{*}{ Fuente de variabilidad } & \multicolumn{5}{|c|}{ Velocidad de onda en $F$. americana } \\
\hline & $\sigma$ & $\%_{\text {var }}$ & $\sigma^{2}$ & $\%_{\text {con }}$ & $\% \mathrm{R} \& \mathrm{R}$ \\
\hline Repetibilidad & 164 & 38 & 26987 & 15 & 16 \\
\hline Reproducibilidad & 376 & 88 & 141070 & 77 & 81 \\
\hline Interacción & 72 & 17 & 5202 & 3 & 3 \\
\hline $\mathrm{R} \& \mathrm{R}$ & 416 & 97 & 173258 & 94 & 100 \\
\hline Probetas & 102 & 24 & 10382 & 6 & \\
\hline Variación total & 429 & 100 & 183640 & & \\
\hline \multirow[t]{2}{*}{ Fuente de variabilidad } & \multicolumn{5}{|c|}{ Velocidad de onda en $A$. plurijuga } \\
\hline & $\sigma$ & $\%_{\text {var }}$ & $\sigma^{2}$ & $\%_{\text {con }}$ & $\% \mathrm{R} \& \mathrm{R}$ \\
\hline Repetibilidad & 422 & 64 & 178258 & 40 & 44 \\
\hline Reproducibilidad & 478 & 72 & 228514 & 52 & 56 \\
\hline Interacción & 0 & 0 & 0 & 0 & 0 \\
\hline $\mathrm{R} \& \mathrm{R}$ & 638 & 96 & 406771 & 92 & 100 \\
\hline Probetas & 184 & 28 & 33951 & 8 & \\
\hline Variación total & 664 & 100 & 440723 & & \\
\hline
\end{tabular}

Dos operadores por instrumento efectuando dos mediciones en 20 partes (probetas). $\sigma=$ Desviación estándar; $\%_{\text {var }}=$ Porcentaje variación total; $\sigma^{2}=$ Varianza; $\%_{\text {con }}=$ Porcentaje de contribución; $\% R \& R=$ Contribución a la repetibilidad y reproducibilidad. Fuente: elaboración propia.

Tabla 3. Resultados del análisis de varianza de los estudios de repetibilidad y reproducibilidad

\begin{tabular}{|l|l|l|l|l|l|}
\hline Fuente de variabilidad & \multicolumn{5}{|c|}{ Velocidad de onda en F. americana } \\
\hline & SC & GL & CM & Razón F & Valor P \\
\hline Instrumentos (2) & 5680180 & 1 & 5680180 & 152 & $<0.0001 \#$ \\
\hline Probetas (20) & 1499470 & 19 & 78919 & 2 & $0.0560^{*}$ \\
\hline Instrumentos $\times$ probetas & 710401 & 19 & 37390 & 1 & $0.1890^{*}$ \\
\hline Error residual & 1079460 & 40 & 26987 & & \\
\hline Total & 8969510 & 79 & & & \\
\hline Fuente de variabilidad & & \multicolumn{2}{|c|}{ Velocidad de onda en A. plurijuga } & \\
\hline & SC & GL & CM & Razón F & Valor P \\
\hline Instrumentos (2) & 9154400 & 1 & 9154400 & 661 & $<0.0001 \#$ \\
\hline Probetas (20) & 2843590 & 19 & 149663 & 11 & $<0.0001 \#$ \\
\hline Instrumentos $\times$ probetas & 263284 & 19 & 13857 & 0 & $1.0000^{*}$ \\
\hline Error residual & 7130320 & 40 & 178258 & & \\
\hline Total & 19391600 & 79 & & & \\
\hline
\end{tabular}

Dos operadores por instrumento efectuando dos mediciones en 20 partes (probetas). $\mathrm{SC}=$ Suma de cuadrados; $\mathrm{GL}=$ Grados de libertad; $\mathrm{CM}=$ Cuadrado medio; ${ }^{*} \mathrm{P}_{(\alpha=0,05)}>0,05=$ No existe interacción significativa; $\# \mathrm{P}_{(\alpha=0,05)}<0,05=$ Sí existe interacción significativa. Fuente: elaboración propia. 
rijuga y solamente de esta especie) con un instrumento de medición (Metriguard y solamente este aparato; o comparativamente, Sylvatest $t^{\circledR}$ y solamente este aparato). Por su parte, la repetividad para $F$. americana contribuye a la variabilidad total un $15 \%$ y para $A$. plurijuga un $40 \%$.

No obstante que las mediciones de las velocidades de onda son precisas con coeficientes de variación máximos de $10 \%$, se observan diferencias entre especies. Una causa que provoca la variabilidad de la velocidad de onda en la madera son las diferentes configuraciones de las pruebas con que se miden (Bachtiar et al., 2017). Si bien los parámetros medidos son teóricamente equivalentes, no lo son sus solicitaciones. Una interpretación de estos resultados implica considerar que cada uno de los dos instrumentos mide el tiempo de transmisión de la onda en la dirección longitudinal de la probeta con diferentes tipos de solicitaciones. Las ondas de esfuerzo provocan la vibración de la masa de una sección transversal al sentido de la fuerza que origina el movimiento. En cambio, las ondas de ultrasonido viajan en paquete a través de la estructura material de la madera.

La reproducibilidad representa la variación causada por los operadores, más la combinación de operadores y probetas. En esta investigación se trata de mediciones en la misma especie ( $F$. americana o $A$. plurijuga) pero con diferentes instrumentos (Metriguard ${ }^{\oplus}$ y Sylvatest ${ }^{\oplus}$ a la vez). La reproductibilidad contribuye a la variabilidad total para $F$. americana $77 \%$ y para A. plurijuga 52\%. Este resultado es mayor a la contribución adjudicada a los instrumentos (repetibilidad). En efecto, los resultados sugieren que la variabilidad es específica a cada especie $y$, de esta manera, se distinguen sus características mecánicas para fines de diseño y cálculo ingenieril.

La interacción representa la variación causada por la combinación de operadores y pro- betas. Para F. americana, la contribución de la interacción es de 3\%. En cambio, para $A$. plurijuga el porcentaje de contribución a la variabilidad total es nulo (Tabla 2). Este resultado sugiere que los operadores midieron las probetas con precisión y exactitud. Particularmente en el caso de las velocidades de onda en la madera de A. plurijuga.

La contribución a la variabilidad total de R\&R de $F$. americana es $94 \%$ y para $A$. plurijuga es 92\% (Tabla 2). De acuerdo con Woodall y Borror (2008) y Yeh y Sun (2013), en los estudios de R\&R enfocados al análisis de sistemas de medición, los valores superiores al $30 \%$ generalmente se consideran inaceptables y se recomienda hacer todo lo posible para mejorar el sistema de medición. Este criterio va más allá de los alcances de esta investigación. Empero, los resultados del diseńo experimental en lo concerniente a las velocidades de onda indican normalidad de las distribuciones y suficiencia en el tamaño de las muestras estudiadas (Tabla 1). Asimismo, la verificación de varianzas indica que no existen diferencias estadísticamente significativas entre las desviaciones estándar (con un nivel del 95\% de confianza), mientras que el análisis de varianza distingue entre muestras de acuerdo con el instrumento de medición ( $M e-$ triguard ${ }^{\oplus}$ versus Sylvatest ${ }^{\oplus}$ ).

La contribución a la variabilidad total adjudicada a las probetas para $F$. americana es de 6\% y para $A$. plurijuga de $8 \%$ (Tabla 2 ). Estos resultados pueden compararse con los coeficientes de variación (Tabla 1 ) de ondas de esfuerzo y ultrasonido: para $F$. americana son en promedio de $5 \%$ y para $A$. plurijuga son en promedio de $8 \%$, magnitudes prácticamente equivalentes. En el mismo contexto, los valores promedio de las velocidades de onda de ultrasonido para $F$. americana son $13 \%$ mayores con respecto a las de ultrasonido y para $A$. plurijuga este cálculo es de $17 \%$. Estas diferencias, según la técnica em- 
pleada, han sido observadas en otras especies y con los métodos aquí utilizados por Sotomayor (2014) y Sotomayor (2015). Estos resultados resaltan las ventajas de los estudios de $\mathrm{R} \& \mathrm{R}$ respecto al análisis tradicional que presenta la literatura en ciencias y tecnología de la madera. Mientras que la Tabla 1 provee únicamente los coeficientes de variación como medida para estimar la precisión de las mediciones, los datos de las Tablas 2 y 3 indican la variabilidad de los instrumentos, operadores y probetas, así como su interacción, que en este caso coincide con el promedio de los coeficientes de variación de cada muestra.

En síntesis, los resultados pueden explicarse por la variabilidad natural en la magnitud de las características físicas que existe entre especies y al interior de una especie (Perré et al., 2016), por la heterogeneidad anatómica de las maderas (Schubert et al., 2009), por la anisotropía material (Brémaud et al., 2011) y por la higroscopicidad del tejido leñoso (Mvondo et al., 2017). La diversidad en las propiedades mecánicas de la madera también depende de factores genéticos $y$ ambientales y se encuentra en todos los niveles tanto entre especies gimnospermas y angiospermas, como entre árboles de climas tropicales y templados, entre poblaciones de una especie determinada, entre árboles de una población y finalmente, entre ubicaciones de muestreo dentro de un solo árbol (Zhang et al. 2011). Sin embargo, el diseño experimental y los estudios de $\mathrm{R} \& \mathrm{R}$ mostraron de manera explícita los diferentes componentes de la variabilidad total en las magnitudes de las velocidades de onda para F. americana y A. plurijuga.

\section{Conclusiones}

Los estudios de $\mathrm{R} \& \mathrm{R}$ de las velocidades de onda determinadas en maderas de $F$. americana y $A$. plurijuga, con instrumentos de ondas de esfuerzo y de ultrasonido, permiten cuantificar las fuentes de variabilidad entre los instrumentos, los operadores y las probetas. La investigación informa sobre la conveniencia de los estudios de $R \& R$ para la validación de las mediciones de velocidades de onda con los dos métodos citados. La contribución a la variabilidad entre los dos instrumentos de medición es pequeña en comparación con la variabilidad entre las especies $F$. americana y $A$. plurijuga.

La variabilidad en la velocidad de onda medida en una probeta es causada principalmente por el instrumento de medición. Las magnitudes de las interacciones son particulares a cada caso de estudio. Los resultados están restringidos por la experimentación de muestras homogéneas y estadísticamente representativas de las maderas estudiadas y son válidos para las condiciones particulares de la metodología empleada.

\section{Agradecimientos}

La investigación estuvo patrocinada por la Universidad Michoacana de San Nicolás de Hidalgo (UMSNH) en Morelia, Michoacán, México. Se agradece la participación en las mediciones de laboratorio a los alumnos de la Facultad de Ingeniería en Tecnología de la Madera, de la UMSNH.

\section{Referencias}

Bachtiar, E. V., Sanabria, S. J., Mittig, J. P., y Nimes, P. 2017. Moisture-dependent elastic characteristics of walnut and cherry wood by means of mechanical and ultrasonic test incorporating three different ultrasound data evaluation techniques. Wood Science and Technology, 51 (1): 47-67.

Botero, M., Arbeláez, O., y Mendoza, J. A. 2007. Método ANOVA utilizado para realizar el estudio de repetibilidad y reproducibilidad dentro del control de calidad de un sistema de medición. Scientia et Technica, 13 (37): 533-537. 
Brémaud, I., Gril, J., y Thibaut, B. (2011). Anisotropy of wood vibrational properties: dependence on grain angle and review of literature data. Wood Science and Technology, 45 (4): 735-754.

De Mast, J., y Trip, A. 2005. Gauge R\&R Studies for Destructive Measurements. Journal of Quality Technology, 37(1): 40-49.

De Oliveira, F. G. R., y Sales, A. 2006. Relationship between density and ultrasonic velocity in Brazilian tropical Woods. Bioresource Technology, 97 (18): 2443-2446.

Del Menezzi, C. H. S., Amorim, M. R. S., Costa, M. A., y Garcez, L. R. O. 2014. Evaluation of Thermally Modified Wood by Means of Stress Wave and Ultrasound Nondestructive Methods. Materials Science (Medziagotyra), 20 (1): 61-66.

Gómez, R. A. 2013. Modelo estadístico de medición $\mathrm{R} \& \mathrm{R}$ en el pesaje de productos de la preparación de pedidos en un centro de distribución. Revista Soluciones de Postgrado EIA, 5 (10): 45-64.

Gonçalves, R. A., Trinca, J., y Pellis, B. P. 2014. Elastic constants of wood determined by ultrasound using three geometries of specimens. Wood Science and Technology, 48 (2): 269-287.

Gong, L., Burdick, R. K., y Quiroz, J. 2015. Confidence Intervals for Unbalanced Two-factor Gauge R\&R Studies. Quality and Reliability Engineering International, 21: 727-741.

GonzÁlez, G. y Falcón, C. F. 2015. Procedimiento para el análisis de repetibilidad y reproducibilidad en procesos de manufactura. Revista Cubana de Ingeniería, 6 (3): 53-59.

Gutiérrez, H., y De la Vara, R. 2009. Análisis y diseño de experimentos. México: McGraw-Hill.

Gutiérrez, H., y De la Vara, R. 2013. Control estadístico de la calidad y Seis Sigma. México: Mc Graw Hill.

HA, C., Kim, D. S., y Park, S. 2017. Assessment of the Adequacy of Gauge Repeatability and Reproducibility Study Using a Monte Carlo Simulation. Mathematical Problems in Engineering, 2017 (7237486): 1-15.

Hasegawa, M., Takata, M., Matsumura, J., y Oda, K. 2011. Effect of wood properties on within-tree variation in ultrasonic wave velocity in softwood. Ultrasonics, 51 (3): 296-302.

INTERNATIONAL Organization for Standardization. 2012 . ISO 3129:2012. Wood. Sampling methods and general requirements for physical and mechanical testing of small clear wood specimens. Geneva: International Organization for Standardization.

INTERNATIONAL Organization for Standardization. 2014a. ISO 13061-2:2014. Physical and mechani- cal properties of wood. Test methods for small clear wood specimens. Part 2: Determination of density for physical and mechanical tests. Geneva: International Organization for Standardization.

INTERNATIONAL Organization for Standardization. 2014b. ISO 13061-1:2014. Physical and mechanical properties of wood. Test methods for small clear wood specimens. Part 1: Determination of moisture content for physical. Geneva: International Organization for Standardization.

LI, M. C., y Al-Refaie, A. 2008. Improving Wooden Parts' Quality by Adopting DMAIC Procedure. Quality and Reliability Engineering International, 24: 351-360.

Lin, W. y Wu. J. 2013. Nondestructive Testing of Wood Defects based on Stress Wave Technology. Telkomnika, 11 (11): 6802-6807.

López, G., Mazaira, Z., Hernández, I. A., y Cabrera, H. R. 2018. Análisis de la calidad de las mediciones en el proceso de laboratorio. Revista Espacios, 39 (6): 1-18.

Mohamed, O. A., Masood, S. H., y Bhowmik, J. L. 2018. Investigation of dimensional variation in parts manufactured by fused deposition modeling using Gauge Repeatability and Reproducibility. IOP Conf. Series: Materials Science and Engineering, 310: 012090.

Mendes, L. M., Gomes, R., Santana, R., Rotela, P., y De Freitas, J. H. 2019. New indicators for measurement error detection in GR\&R studies. Measurement, 140: 557-564.

Mvondo, R. R. N., Meukam, P., Jeong, J., Meneses, D. D., y Nkeng, E.G. 2017. Influence of water content on the mechanical and chemical properties of tropical wood species. Results in Physics, 7: 2096-2103.

Nasir, V., Nourian, S., Avramidis, S., y Cool, J. 2019. Stress wave evaluation by accelerometer and acoustic emission sensor for thermally modified wood classification using three types of neural networks. European Journal of Wood and Wood Products, 77 (1): 45-55.

Perré, P., Almeida, G., Ayouz, M., y Frank, X. 2016. New modelling approaches to predict wood properties from its cellular structure: image-based representation and meshless methods. Annals of Forest Science, 73 (1): 147-162.

Peruchi, R. S., Balestrassi, P. P., De Paiva, A. P., Ferreira, J. R., y Carmelossi, M. S. 2013. A new multivariate gage $R \& R$ method for correlated characteristics. International Journal of Production Economics, 144 (1): 301-315.

SaIKAEW, C. 2018. An implementation of measurement system analysis for assessment of machine and part 
variations in turning operation. Measurement, 118: 246-252.

Schubert, S., Gsell, D., Dual, J., Motavalli, M., y Niemz, P. 2009. Acoustic wood tomography on trees and the challenge of wood heterogeneity. Holzforschung, 63 (1): 107-112.

Senol, S. 2004. Measurement system analysis using designed experiments with minimum $\alpha-\beta$ risks and $n$. Measurement, 36: 131-141.

Senvar, O. y First, S. U. K. 2010. An overview of capability evaluation of Measurement Systems and Gauge Repeatability and Reproducibility Studies. International Journal of Metrology and Quality Engineering, 1 (2): 121-127.

SHI, L., Chen, W., y Lu, L. F. 2014. An Approach for Simple Linear Profile Gauge R\&R Studies. Discrete Dynamics in Nature and Society, 2014(816980): $1-7$.

Sotomayor, J. R. 2014. Caracterización mecánica de la madera con métodos no destructivos. Morelia: Universidad Michoacana de San Nicolás de Hidalgo.

Sotomayor, J. R. 2015. Banco FITECMA de características físico-mecánicas de maderas mexicanas. Morelia: Universidad Michoacana de San Nicolás de Hidalgo.

Sotomayor, J. R. 2020. Efecto del preservado de la madera con boro sobre el módulo dinámico. Evaluación por medio de ultrasonido. Científica, 24 (1): 67-76.

Sotomayor, J. R., y Ávila, L. E. A. 2020a. Efecto de la impregnación con boro sobre las densidades y módulos dinámicos de tres maderas angiospermas. Revista Ingeniería al Día, en prensa.

SotomaYor, J. R., y Ávila, L. E. A. 2020b. Impregnación con sales de boro de la madera de Spathodea campanulata P. Beauv, Fraxinus americana L. y Albizia plurijuga (Standl.) Britton y Rose. Evaluación con ultrasonido de la velocidad de onda y del módulo dinámico. Revista Internacional de Investigación e Innovación Tecnológica, 8 (45): 40-50.

Sotomayor, J. R. y Correa, S. 2016. Retención de sales de boro en la madera y su efecto en el módulo de elasticidad dinámico. Revista Científica, 24 (1): 1-19.
Sotomayor, J. R., Adachi, K., Iida, R., y Hayashi, T. 2020. Aumento del módulo dinámico en madera densificada. Evaluación con ondas de esfuerzo en ocho especies. Avances en Investigación Agropecuaria, 24 (1): 7-20.

Sotomayor, J. R., García, L. J., Hernández, S. A., Moya, C. E., y Olguín, J. B. 2011. Dispositivo de usos múltiples para pruebas no destructivas en madera y materiales compuestos de madera. Ultrasonido, Ondas de esfuerzo y Vibraciones transversales. Investigación e Ingeniería de la Madera, 7 (3): 20-33.

Tiplica, T., Verron, S., Grémy-Gros, C., Vandewalle, P., y Mehinagic, E. 2015. On the quality of acoustical measures when evaluating fruits quality. International Journal of Metrology and Quality Engineering, 6 (201): 1-8.

WANG, F. K. y Chien, T. W. 2010. Process-oriented basis representation for a multivariate gauge study. Computers y Industrial Engineering, 58: 143-150.

Woodall, W. H. y Borror, C. M. 2008. Some Relationships between Gage R\&R Criteria. Quality and Reliability Engineering International, 24: 99-106.

YeH, T., y Sun, J. 2013. Using the Monte Carlo Simulation Methods in Gauge Repeatability and Reproducibility of Measurement System Analysis. Journal of Applied Research and Technology, 11 (5): 780-796.

Yu, Y., Liu, Y., Gong, M., Xu, Z., y Fang, Y. 2017. R\&R study of using a stress wave timer to measure the elastic modulus of structural dimension lumber. Measurement, 95: 293-296.

Zanobini, A., Sereni, B., Catelani, M., y Ciani, L. 2016. Repeatability and Reproducibility techniques for the analysis of measurement systems. Measurement, 86: 125-132.

Zhang, T., Bai, S. L., Bardet, S., Alméras, T., Thibaut, B., y Beauchêne, J. 2011. Radial variations of vibrational properties of three tropical woods. Journal of Wood Science, 57 (5): 377-386. 